Gazi University
Journal of Science
$\mathrm{http} / /$ dergipark.gov.tr/gujs

\title{
Well-Defined Solutions of a Three-Dimensional System of Difference Equations
}

\author{
Merve KARA $^{1, *(D)}$, Nouressadat TOUAFEK ${ }^{2}$ (D) Yasin YAZLIK $^{3}$ \\ ${ }^{1}$ Aksaray University, Ortaköy Vocational High School, 68400, Aksaray, Turkey \\ ${ }^{2}$ Mohamed Seddik Ben Yahia University, LMAM Laboratory, Department of Mathematics, 18000, Jijel, Algeria \\ ${ }^{3}$ Nevsehir Haci Bektas Veli University, Faculty of Science and Art, Department of Mathematics, 50300, Nevsehir, Turkey
}

\section{Highlights}

- This paper focuses on a three-dimensional system of difference equations.

- The three-dimensional system of difference equations were solved.

- Asymptotic behavior of solutions were determined and periodic solutions were demonstrated.

\section{Article Info}

Received: 01/11/2019

Accepted: 24/04/2020

\section{Keywords}

Periodic solutions Difference equations, Three-dimensional systems of difference equations

\begin{abstract}
We show that the three-dimensional system of difference equations

$x_{n+1}=\frac{a x_{n} z_{n-1}}{z_{n}-\beta}+\gamma, y_{n+1}=\frac{b y_{n} x_{n-1}}{x_{n}-\gamma}+\alpha, z_{n+1}=\frac{c z_{n} y_{n-1}}{y_{n}-\alpha}+\beta, n \in N_{0}$,

where the parameters $a, b, c, \alpha, \beta, \gamma$ and the initial conditions $x_{-i}, y_{-i}, z_{-i}, i \in\{0,1\}$, are nonzero real numbers, can be solved. Using the obtained formulas, we determine the asymptotic behavior of solutions and give conditions for which periodic solutions exist. Some numerical examples are given to demonstrate the theoretical results.
\end{abstract}

\section{INTRODUCTION}

Difference equations and their systems are related to many real life models in different branches of modern science such as biology, physics, economics, etc, in $[1,2]$. This is due to the fact that these models are expressed by discrete equations and this explain why difference equations and their systems have attracted the attention of many researchers in recent years in [3-6]. One of the most popular subject associated with difference equations and their system, especially the non-linear ones, is to examine their solvability and the behavior of their solutions in [7-35]. Also in these references, we can find several generalizations of solvable difference equations and systems in different ways by adding parameters, increasing the order, increasing dimensional, as in the examples given below: In an earlier paper, Elabbasy et al., in [36], dealt with, among other things, the following difference equation

$$
x_{n+1}=\frac{x_{n} x_{n-1}}{x_{n}-1}+1, n \in N_{0} .
$$

Then, in [37], Equation (1) was extended to the following two-dimensional system of difference equations

$$
x_{n+1}=\frac{a x_{n} y_{n-1}}{y_{n}-\alpha}+\beta, y_{n+1}=\frac{b y_{n} x_{n-1}}{x_{n}-\beta}+\alpha, n \in N_{0},
$$


where the parameters $a, b, \alpha, \beta$ and the initial values $x_{-i}, y_{-i}, i=0,1$, are non-zero real numbers. They obtained the forms of solutions of system (2) and investigated their behavior. Quite recently, in [38], Equation (1) and System (2) were generalized to the following difference equations system by both increasing order and taking periodic coefficients

$x_{n+1}=\frac{a_{n} x_{n-k+1} y_{n-k}}{y_{n}-\alpha_{n}}+\beta_{n+1}, y_{n+1}=\frac{b_{n} y_{n-k+1} x_{n-k}}{x_{n}-\beta_{n}}+\alpha_{n+1}, n \in N_{0}$,

where the sequences $\left(a_{n}\right)_{n \in N_{0}},\left(b_{n}\right)_{n \in N_{0}},\left(\alpha_{n}\right)_{n \in N_{0}},\left(\beta_{n}\right)_{n \in N_{0}}$ are two periodic and the initial conditions $x_{-i}, y_{-i}, i=\overline{0, k}$, are non-zero real numbers. Also, by using obtained formulas they give the asymptotic behavior and studied the periodicity of well-defined solutions of system (3) when $a_{0}=b_{1}$ and $a_{1}=b_{0}$.

A natural question is if any of the corresponding three-dimensional relatives to Equation (1), Systems (2) and (3) is also solvable. Here we give a positive answer to this question, by proving that the following threedimensional system

$x_{n+1}=\frac{a x_{n} z_{n-1}}{z_{n}-\beta}+\gamma, y_{n+1}=\frac{b y_{n} x_{n-1}}{x_{n}-\gamma}+\alpha, z_{n+1}=\frac{c z_{n} y_{n-1}}{y_{n}-\alpha}+\beta, n \in N_{0}$,

where the parameters $a, b, c, \alpha, \beta, \gamma$ and the initial values $x_{-i}, y_{-i}, z_{-i}, i=0,1$, are non-zero real numbers, is solvable in closed form.

The following well known result, see for example [39], will be very useful in our work.

Lemma 1. Let $a, b$ be two real numbers and consider the first order linear difference equation

$$
y_{n+1}=a y_{n}+b, n \in N_{0} \text {. }
$$

Then the sequence $\left(y_{n}\right)_{n \in N_{0}}$, the solution of equation (5), has the form

$y_{n}= \begin{cases}a^{n} y_{0}+b\left(\frac{1-a^{n}}{1-a}\right), & \text { if } a \neq 1, \\ y_{0}+b n, & \text { if } a=1 .\end{cases}$

Definition 1. (Periodicity). A sequence $\left(x_{n}\right)_{n-k}^{\alpha}$ is said to be eventually periodic with period $p$ if there exist $n_{0} \geq-k$ such that $x_{n+p}=x_{n}$ for all $n \geq n_{0}$. If $n_{0}=-k$, then the sequence $\left(x_{n}\right)_{n-k}^{\infty}$ is said to be periodic with period $p$.

Throughout this paper we suppose that $\prod_{j=i}^{m} A_{j}=1$ and $\sum_{j=i}^{m} A_{j}=0$, for all $m<i$.

\section{SOLUTIONS OF SYSTEM (4)}

Let $\left\{\left(x_{n}, y_{n}, z_{n}\right)\right\}_{n \geq-1}$ be well-defined solutions of system (4), that is the solutions such that $x_{n} \neq \gamma, y_{n} \neq$ $\alpha, z_{n} \neq \beta$, for all $n \in N_{0}$. If one of the initial values $x_{-j}, y_{-j}, z_{-j}, j=0,1$, is equal to zero, then the corresponding solutions of system (4) is not defined. For example, if $x_{-1}=0$, then $y_{1}=\alpha$, and so $z_{2}$ can not be calculated. Now assume that $x_{-j} \cdot y_{-j} \cdot z_{-j} \neq 0, j=0,1$. If one of the terms $x_{n_{0}}, y_{n_{0}}$ and $z_{n_{0}}$ for $n_{0} \geq 1$, is equal to zero, then from system (4) either $x_{n_{0}+1}=\frac{a x_{n_{0}} z_{n_{0}-1}}{z_{n_{0}}-\beta}+\gamma=\gamma$ and so, it follows that, $y_{n_{0}+2}$ is not defined or $y_{n_{0}+1}=\frac{b y_{n_{0}} x_{n_{0}-1}}{x_{n_{0}}-\gamma}+\alpha=\alpha$ and so, it follows that, $z_{n_{0}+2}$ is not defined or $z_{n_{0}+1}=$ $\frac{c z_{n_{0}} y_{n_{0}-1}}{y_{n_{0}}-\alpha}+\beta=\beta$ and so, it follows that, $x_{n_{0}+2}$ is not defined. Thus, well-defined solutions of system (4) satisfy $x_{n} \cdot y_{n} \cdot z_{n} \neq 0, n \geq-1$. By means of the change of variables

$$
u_{n}=\frac{x_{n}-\gamma}{x_{n-1}}, v_{n}=\frac{y_{n}-\alpha}{y_{n-1}}, w_{n}=\frac{z_{n}-\beta}{z_{n-1}}, n \in N_{0},
$$


the system in (4) becomes

$$
u_{n+1}=\frac{a}{w_{n}}, v_{n+1}=\frac{b}{u_{n}}, w_{n+1}=\frac{c}{v_{n}}, n \in N_{0} .
$$

From (7), we have three independent equations

$$
u_{n+6}=u_{n}, v_{n+6}=v_{n}, w_{n+6}=w_{n}, n \in N_{0}
$$

that is the sequences $\left(u_{n}\right)_{n \in N_{0}},\left(v_{n}\right)_{n \in N_{0}}$ and $\left(w_{n}\right)_{n \in N_{0}}$ are periodic of period 6. So,

$$
u_{6 n+j}=u_{j}, v_{6 n+j}=v_{j}, w_{6 n+j}=w_{j}, j=\overline{0,5}, n \in N_{0},
$$

From (6) we have that

$$
x_{n}=u_{n} x_{n-1}+\gamma, y_{n}=v_{n} y_{n-1}+\alpha, z_{n}=w_{n} z_{n-1}+\beta, n \in N_{0} .
$$

Equations in (8) and (10) can be written in the form

$$
\begin{aligned}
& x_{6 n}=u_{6 n} x_{6 n-1}+\gamma=u_{0} x_{6 n-1}+\gamma, n \in N_{0}, \\
& x_{6 n+1}=u_{6 n+1} x_{6 n}+\gamma=u_{1} x_{6 n}+\gamma, n \in N_{0}, \\
& x_{6 n+2}=u_{6 n+2} x_{6 n+1}+\gamma=u_{2} x_{6 n+1}+\gamma, n \in N_{0}, \\
& x_{6 n+3}=u_{6 n+3} x_{6 n+2}+\gamma=u_{3} x_{6 n+2}+\gamma, n \in N_{0}, \\
& x_{6 n+4}=u_{6 n+4} x_{6 n+3}+\gamma=u_{4} x_{6 n+3}+\gamma, n \in N_{0}, \\
& x_{6 n+5}=u_{6 n+5} x_{6 n+4}+\gamma=u_{5} x_{6 n+4}+\gamma, n \in N_{0}, \\
& x_{6 n+6}=u_{6 n+6} x_{6 n+5}+\gamma=u_{0} x_{6 n+5}+\gamma, n \in N_{0}, \\
& y_{6 n}=v_{6 n} y_{6 n-1}+\alpha=v_{0} y_{6 n-1}+\alpha, n \in N_{0}, \\
& y_{6 n+1}=v_{6 n+1} y_{6 n}+\alpha=v_{1} y_{6 n}+\alpha, n \in N_{0}, \\
& y_{6 n+2}=v_{6 n+2} y_{6 n+1}+\alpha=v_{2} y_{6 n+1}+\alpha, n \in N_{0}, \\
& y_{6 n+3}=v_{6 n+3} y_{6 n+2}+\alpha=v_{3} y_{6 n+2}+\alpha, n \in N_{0}, \\
& y_{6 n+4}=v_{6 n+4} y_{6 n+3}+\alpha=v_{4} y_{6 n+3}+\alpha, n \in N_{0}, \\
& y_{6 n+5}=v_{6 n+5} y_{6 n+4}+\alpha=v_{5} y_{6 n+4}+\alpha, n \in N_{0}, \\
& z_{6 n+6}=v_{6 n+6} y_{6 n+5}+\alpha=v_{0} y_{6 n+5}+\alpha, n \in N_{0}, \\
& z_{6 n+2}=w_{6 n} z_{6 n-1}+\beta=w_{6 n+1} z_{6 n}+\beta=w_{6 n+2} z_{6 n}+\beta, n \in N_{0}, \\
& z_{6 n+1}+\beta=w_{2} z_{6 n+1}+\beta, n \in N_{0},
\end{aligned}
$$




$$
\begin{aligned}
& z_{6 n+3}=w_{6 n+3} z_{6 n+2}+\beta=w_{3} z_{6 n+2}+\beta, n \in N_{0}, \\
& z_{6 n+4}=w_{6 n+4} z_{6 n+3}+\beta=w_{4} z_{6 n+3}+\beta, n \in N_{0}, \\
& z_{6 n+5}=w_{6 n+5} z_{6 n+4}+\beta=w_{5} z_{6 n+4}+\beta, n \in N_{0}, \\
& z_{6 n+6}=w_{6 n+6} z_{6 n+5}+\beta=w_{0} z_{6 n+5}+\beta, n \in N_{0},
\end{aligned}
$$

which implies that

$$
\begin{aligned}
& x_{6(n+1)+j}=\left(\prod_{i=0}^{5} u_{i}\right) x_{6 n+j}+\gamma \sum_{s=1}^{6}\left(\prod_{i=s+1}^{6} u_{(j+i) \bmod (6)}\right), \\
& y_{6(n+1)+j}=\left(\prod_{i=0}^{5} v_{i}\right) y_{6 n+j}+\alpha \sum_{s=1}^{6}\left(\prod_{i=s+1}^{6} v_{(j+i) \bmod (6)}\right), \\
& z_{6(n+1)+j}=\left(\prod_{i=0}^{5} w_{i}\right) z_{6 n+j}+\beta \sum_{s=1}^{6}\left(\prod_{i=s+1}^{6} w_{(j+i) \bmod (6)}\right) .
\end{aligned}
$$

For $n \in N_{0}, j=\overline{0,5}$. Let,

$K_{n}^{(j)}=x_{6 n+j}, L_{n}^{(j)}=y_{6 n+j}, M_{n}^{(j)}=z_{6 n+j}, n \in N_{0}, j=\overline{0,5}$.

Then from (11)-(13), we get the following first order linear difference equations with constants coefficients

$K_{n+1}^{(j)}=A K_{n}^{(j)}+B^{(j)}, L_{n+1}^{(j)}=C L_{n}^{(j)}+D^{(j)}, M_{n+1}^{(j)}=E M_{n}^{(j)}+F^{(j)}, n \in N_{0}, j=\overline{0,5}$,

where

$$
\begin{aligned}
& A=\prod_{i=0}^{5} u_{i}, B^{(j)}=\gamma \sum_{s=1}^{6}\left(\prod_{i=s+1}^{6} u_{(j+i) \bmod (6)}\right), \\
& C=\prod_{i=0}^{5} v_{i}, D^{(j)}=\alpha \sum_{s=1}^{6}\left(\prod_{i=s+1}^{6} v_{(j+i) \bmod (6)}\right), \\
& E=\prod_{i=0}^{5} w_{i}, F^{(j)}=\beta \sum_{s=1}^{6}\left(\prod_{i=s+1}^{6} w_{(j+i) \bmod (6)}\right) .
\end{aligned}
$$

Solving Equations (15) using Lemma 1 and taking in account Equations (14), we obtain for $n \in N_{0}$ and $j=\overline{0,5}$

$$
\begin{aligned}
& x_{6 n+j}=\left\{\begin{array}{cc}
A^{n} x_{j}+B^{(j)}\left(\frac{1-A^{n}}{1-A}\right) & \text { if } A \neq 1, \\
x_{j}+B^{(j)} n & \text { if } A=1,
\end{array}\right. \\
& y_{6 n+j}=\left\{\begin{array}{cc}
C^{n} y_{j}+D^{(j)}\left(\frac{1-C^{n}}{1-C}\right) & \text { if } C \neq 1, \\
y_{j}+D^{(j)} n & \text { if } C=1,
\end{array}\right.
\end{aligned}
$$

and

$$
z_{6 n+j}=\left\{\begin{array}{cc}
E^{n} z_{j}+F^{(j)}\left(\frac{1-E^{n}}{1-E}\right) & \text { if } E \neq 1, \\
z_{j}+F^{(j)} n & \text { if } E=1 .
\end{array}\right.
$$




\section{PERIODICITY AND ASYMPTOTIC BEHAVIOR OF THE SOLUTIONS OF SYSTEM (4)}

The following results are devoted to the asymptotic behavior and the periodicity of well-defined solutions of system (4).

Theorem 1. Assume that $\left\{\left(x_{n}, y_{n}, z_{n}\right)\right\}_{n \geq-1}$ are well-defined solutions of system (4). Then for $j=\overline{0,5}$ the following statements hold.

a) If $(A-1) x_{j}+B^{(j)} \neq 0$, then we get

$\lim _{n \rightarrow \infty}\left|x_{6 n+j}\right|=\left\{\begin{array}{l}\left|\frac{B^{(j)}}{A-1}\right|,|A|<1, \\ \infty, \quad|A|>1 .\end{array}\right.$

Otherwise, if $(A-1) x_{j}+B^{(j)}=0$ and $A \neq 1$, then $x_{6 n+j}=x_{j}$ for all $n \in N_{0}$, that is the sequence $\left(x_{n}\right)_{n \geq-1}$ is eventually periodic with period 6 and takes the form $\left(x_{n}\right)_{n \geq-1}=$ $\left(x_{-1}, x_{0}, x_{1}, x_{2}, x_{3}, x_{4}, x_{5}, x_{0}, x_{1}, \ldots\right)$.

b) If $B^{(j)} \neq 0$ and $A=1$, then $\left|x_{6 n+j}\right| \rightarrow \infty$, as $n \rightarrow \infty$. Otherwise, if $B^{(j)}=0$ and $A=1$, then $x_{6 n+j}=$ $x_{j}$, for all $n \in N_{0}$, that is the sequence $\left(x_{n}\right)_{n \geq-1}$ is periodic of period 6 and takes the form $\left(x_{n}\right)_{n \geq-1}=$ $\left(x_{-1}, x_{0}, x_{1}, x_{2}, x_{3}, x_{4}, x_{-1}, x_{0}, x_{1}, \ldots\right)$.

c) If $(C-1) y_{j}+D^{(j)} \neq 0$, then we get

$\lim _{n \rightarrow \infty}\left|y_{6 n+j}\right|=\left\{\begin{array}{l}\left|\frac{D^{(j)}}{C-1}\right|,|C|<1 \\ \infty, \quad|C|>1 .\end{array}\right.$

Otherwise, if $(C-1) y_{j}+D^{(j)}=0$ and $C \neq 1$, then $y_{6 n+j}=y_{j}$ for all $n \in N_{0}$, that is the sequence $\left(y_{n}\right)_{n \geq-1}$ is eventually periodic with period 6 and takes the form $\left(y_{n}\right)_{n \geq-1}=$ $\left(y_{-1}, y_{0}, y_{1}, y_{2}, y_{3}, y_{4}, y_{5}, y_{0}, y_{1}, \ldots\right)$.

d) If $D^{(j)} \neq 0$ and $C=1$, then $\left|y_{6 n+j}\right| \rightarrow \infty$, as $n \rightarrow \infty$. Otherwise, if $D^{(j)}=0$ and $C=1$, then $y_{6 n+j}=$ $y_{j}$, for all $n \in N_{0}$, that is the sequence $\left(y_{n}\right)_{n \geq-1}$ is periodic with period 6 and takes the form $\left(y_{n}\right)_{n \geq-1}=$ $\left(y_{-1}, y_{0}, y_{1}, y_{2}, y_{3}, y_{4}, y_{-1}, y_{0}, y_{1}, \ldots\right)$.

e) If $(E-1) z_{j}+F^{(j)} \neq 0$, then we get

$$
\lim _{n \rightarrow \infty}\left|z_{6 n+j}\right|=\left\{\begin{array}{l}
\left|\frac{F^{(j)}}{E-1}\right|,|E|<1, \\
\infty, \quad|E|>1 .
\end{array}\right.
$$

Otherwise, if $(E-1) z_{j}+F^{(j)}=0$ and $E \neq 1$, then $z_{6 n+j}=z_{j}$ for all $n \in N_{0}$, that is the sequence $\left(z_{n}\right)_{n \geq-1}$ is eventually periodic with period 6 and takes the form $\left(z_{n}\right)_{n \geq-1}=\left(z_{-1}, z_{0}, z_{1}, z_{2}, z_{3}, z_{4}, z_{5}, z_{0}, z_{1}, \ldots\right)$.

f) If $F^{(j)} \neq 0$ and $E=1$, then $\left|z_{6 n+j}\right| \rightarrow \infty$, as $n \rightarrow \infty$. Otherwise, if $F^{(j)}=0$ and $E=1$, then $z_{6 n+j}=$ $z_{j}$, for all $n \in N_{0}$, that is the sequence $\left(z_{n}\right)_{n \geq-1}$ is periodic with period 6 and takes the form $\left(z_{n}\right)_{n \geq-1}=\left(z_{-1}, z_{0}, z_{1}, z_{2}, z_{3}, z_{4}, z_{-1}, z_{0}, z_{1}, \ldots\right)$.

\section{Proof.}

We will only prove properties (a) and (b) since the other cases can be dealt with in the same manner.

a) Assume that $(A-1) x_{j}+B^{(j)} \neq 0$. 
Clearly, if $|A|<1$, then $|A|^{n} \rightarrow 0$ as $n \rightarrow \infty$. On the other hand, if $|A|>1$, then $|A|^{n} \rightarrow \infty$ as $n \rightarrow \infty$. From Equation (16), we get

$$
\begin{aligned}
\lim _{n \rightarrow \infty}\left|x_{6 n+j}\right|= & \lim _{n \rightarrow \infty}\left|\frac{(A-1) x_{j}+B^{(j)}}{A-1} A^{n}+\left(\frac{B^{(j)}}{1-A}\right)\right| \\
& =\left|\frac{(A-1) x_{j}+B^{(j)}}{A-1} \lim _{n \rightarrow \infty} A^{n}+\left(\frac{B^{(j)}}{1-A}\right)\right| \\
& =\left\{\begin{array}{ll}
\left|\frac{B^{(j)}}{A-1}\right|,|A|<1 \\
\infty, & |A|>1
\end{array} .\right.
\end{aligned}
$$

Now on the other hand $(A-1) x_{j}+B^{(j)}=0$ and $A \neq 1$. Then we have

$$
\begin{aligned}
x_{6 n+j} & =x_{j} A^{n}+B^{(j)}\left(\frac{A^{n}-1}{A-1}\right) \\
& =x_{j} A^{n}+\left(\frac{A^{n}-1}{A-1}\right)\left(-(A-1) x_{j}\right) \\
& =x_{j} A^{n}-\left(A^{n}-1\right) x_{j} \\
& =x_{j},
\end{aligned}
$$

which completes the proof of (a).

b) Let $A=1$. If $B^{(j)} \neq 0$, then from Equation (16) we get

$x_{6 n+j}=x_{j}+B^{(j)} n$.

Letting $n \rightarrow \infty$ in above equations implies that $\left|x_{6 n+j}\right| \rightarrow \infty$. On the other hand, If $B^{(j)}=0$, then obviously, $x_{6 n+j}=x_{j}+B^{(j)} n=x_{j}+0=x_{j}, \forall n \in N_{0}, j=\overline{0,5}$, which completes the proof of (b).

Corollary 1. Assume that $\left\{\left(x_{n}, y_{n}, z_{n}\right)\right\}_{n \geq-1}$ are well-defined solutions of system (4). Then for $j=\overline{0,5}$, the following statements hold.

a) If $A=-1$ then for all $n \in N_{0}$, we get

$$
\left\{\begin{array}{c}
x_{12 n+j}=x_{j} \\
x_{12 n+6+j}=-x_{j}+B^{(j)}
\end{array}\right.
$$

that is the sequence $\left(x_{n}\right)_{n \geq-1}$ is periodic with period 12 and takes the form

$$
\begin{aligned}
\left(x_{n}\right)_{n \geq-1}= & \left(x_{-1}, x_{0}, x_{1}, x_{2}, x_{3}, x_{4},-x_{-1}+B^{(5)},-x_{0}+B^{(0)},-x_{1}+B^{(1)},-x_{2}+B^{(2)},-x_{3}+\right. \\
& \left.B^{(3)},-x_{4}+B^{(4)}, x_{-1}, x_{0}, \ldots\right) .
\end{aligned}
$$

b) If $C=-1$, then for all $n \in N_{0}$, we get

$$
\left\{\begin{array}{c}
y_{12 n+j}=y_{j} \\
y_{12 n+6+j}=-y_{j}+D^{(j)}
\end{array}\right.
$$


that is the sequence $\left(y_{n}\right)_{n \geq-1}$ is periodic with period 12 and takes the form

$$
\begin{aligned}
\left(y_{n}\right)_{n \geq-1}= & \left(y_{-1}, y_{0}, y_{1}, y_{2}, y_{3}, y_{4},-y_{-1}+D^{(5)},-y_{0}+D^{(0)},-y_{1}+D^{(1)},-y_{2}+D^{(2)},-y_{3}+\right. \\
& \left.D^{(3)},-y_{4}+D^{(4)}, y_{-1}, y_{0}, \ldots\right) .
\end{aligned}
$$

c) If $E=-1$, then for all $n \in N_{0}$, we get

$$
\left\{\begin{array}{c}
z_{12 n+j}=z_{j} \\
z_{12 n+6+j}=-z_{j}+F^{(j)}
\end{array}\right.
$$

that is the sequence $\left(z_{n}\right)_{n \geq-1}$ is periodic with period 12 and takes the form

$$
\begin{aligned}
\left(z_{n}\right)_{n \geq-1}= & \left(z_{-1}, z_{0}, z_{1}, z_{2}, z_{3}, z_{4},-z_{-1}+F^{(5)},-z_{0}+F^{(0)},-z_{1}+F^{(1)},-z_{2}+F^{(2)},-z_{3}+F^{(3)},-z_{4}+\right. \\
& \left.F^{(4)}, z_{-1}, z_{0}, \ldots\right) .
\end{aligned}
$$

\section{Proof.}

We will only prove property (a) since the other cases can be dealt with in the same manner.

a) When $A=-1$, then from Equation (16) we have for $n \in N_{0}$,

$$
x_{6 n+j}=(-1)^{n} x_{j}+\left(\frac{1-(-1)^{n}}{2}\right) B^{(j)} .
$$

Replacing $n$ by $2 n$ and respectively by $2 n+1$ in Equation (19), we get

$x_{12 n+j}=(-1)^{2 n} x_{j}+\left(\frac{1-(-1)^{2 n}}{2}\right) B^{(j)}=x_{j}$,

$x_{12 n+6+j}=(-1)^{2 n+1} x_{j}+\left(\frac{1-(-1)^{2 n+1}}{2}\right) B^{(j)}=-x_{j}+B^{(j)}$

for $n \in N_{0}$.

The following result is a direct consequence of Theorem 1 and Corollary 1.

Corollary 2. Assume that $\left\{\left(x_{n}, y_{n}, z_{n}\right)\right\}_{n \geq-1}$ are well-defined solutions of system (4). Then, the following statements hold.

a) If $A=C=E=-1$, then the solutions are periodic with period 12 .

b) If $A=C=E=1$ and $B^{(j)}=D^{(j)}=F^{(j)}=0$ for $j=\overline{0,5}$, then the solutions are periodic with period 6.

c) If $|A|>1,|C|>1,|E|>1$, then for $n \rightarrow \infty$ we have $\left|x_{6 n+j}\right| \rightarrow \infty,\left|y_{6 n+j}\right| \rightarrow \infty$, $\left|z_{6 n+j}\right| \rightarrow \infty$ for $j=\overline{0,5}$, that is the solutions are unbounded.

\section{NUMERICAL EXAMPLES}

To support our theoretical results, we present numerical examples that represent the solutions of various cases of system (4).

Example 1. Consider the system (4) with the initial values $x_{-1}=2, x_{0}=-12, y_{-1}=50, y_{0}=32$, $z_{-1}=4, z_{0}=-60$ and the parameters, $a=1, b=1, c=-1, \alpha=3, \beta=2, \gamma=7$, the solutions are 
represented as in the following figures.

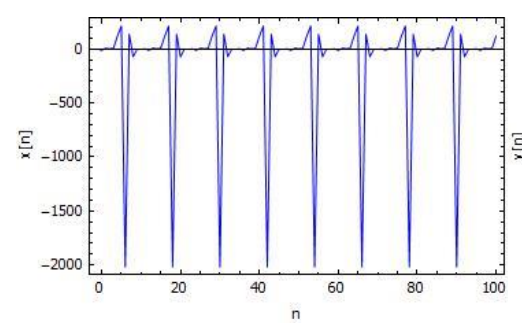

Figure 1. Plots of $x_{n}$

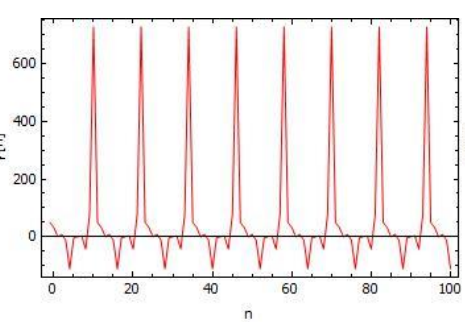

Figure 2. Plots of $y_{n}$

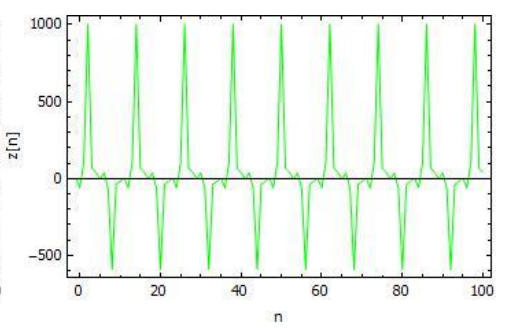

Figure 3. Plots of $z_{n}$

The condition (a) in Corollary 2 is satisfied from Figures (1)- (3). Therefore, the solutions of system (4) are periodic with period 12 .

Example 2. Consider the system (4) with the initial values $x_{-1}=5, x_{0}=4, y_{-1}=-2, y_{0}=7, z_{-1}=$ $3, z_{0}=9$ and the parameters, $a=b=c=1, \alpha=\beta=\gamma=0$, then the solutions are represented as follows:

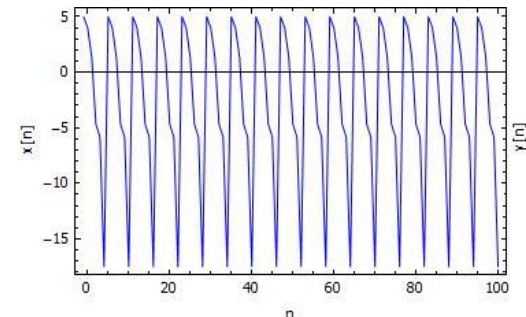

Figure 4. Plots of $x_{n}$

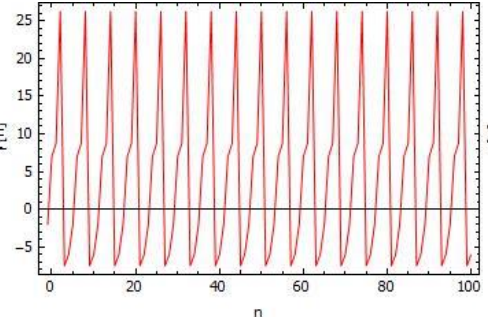

Figure 5. Plots of $y_{n}$

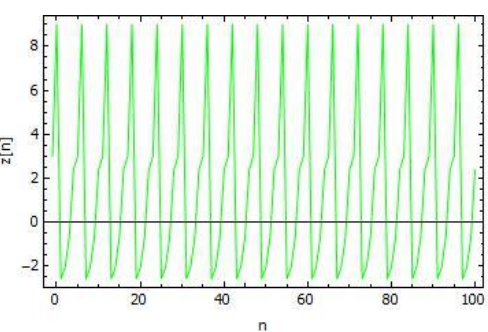

Figure 6. Plots of $z_{n}$

The condition (b) in Corollary 2 is satisfied from Figures (4)-(6). Therefore, the solutions of system (4) are periodic with period 6 .

Example 3. Consider the system (4) with the initial values $x_{-1}=600, x_{0}=700, y_{-1}=80, y_{0}=900$, $z_{-1}=100, z_{0}=110$ and the parameters, $a=2, b=4, c=6, \alpha=7, \beta=2, \gamma=5$, in this case the solutions are represented in the following figures.

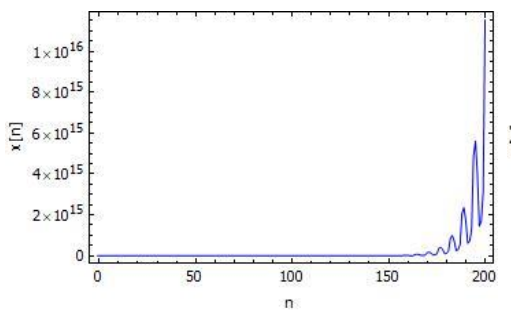

Figure 7. Plots of $x_{n}$

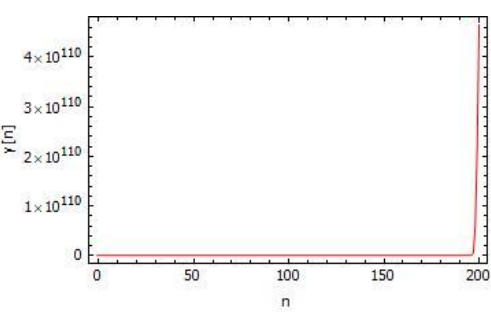

Figure 8. Plots of $y_{n}$

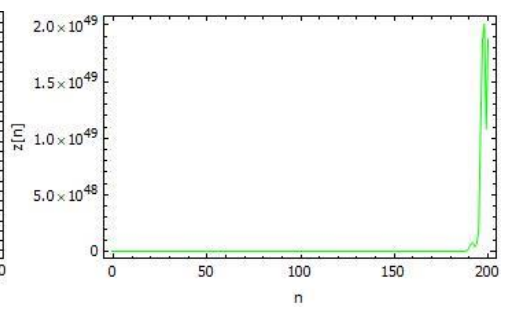

Figure 9. Plots of $z_{n}$

In this case the condition (c) in Corollary 2 is satisfied. From Figures (7)-(9) we can see that $\lim _{n \rightarrow \infty}\left|x_{6 n+j}\right| \rightarrow \infty, \lim _{n \rightarrow \infty}\left|y_{6 n+j}\right| \rightarrow \infty, \lim _{n \rightarrow \infty}\left|z_{6 n+j}\right| \rightarrow \infty$.

Example 4. Consider the system (4) with the initial values $x_{-1}=1.3, x_{0}=0.2, y_{-1}=2.5, y_{0}=0.9$, $z_{-1}=1.8, z_{0}=1.1$ and the parameters, $a=1, b=2, c=5, \alpha=2.6, \beta=1.7, \gamma=0$, the solutions are represented in following figures. 


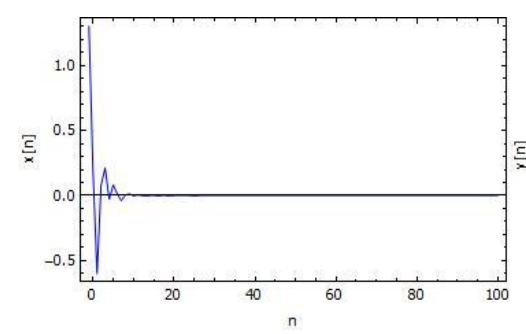

Figure 10. Plots of $x_{n}$

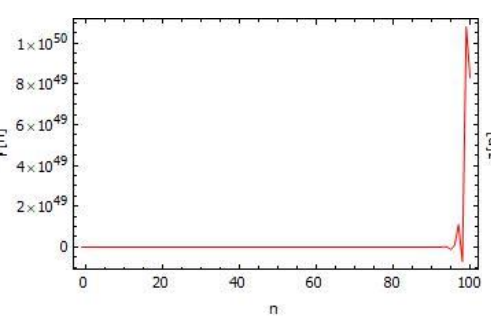

Figure 11. Plots of $y_{n}$

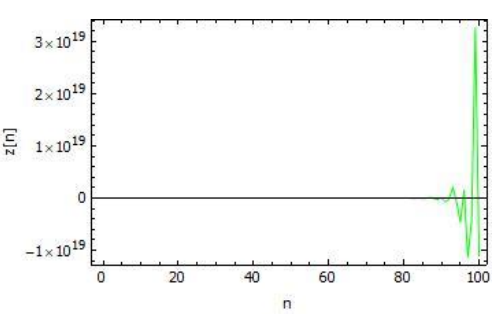

Figure 12. Plots of $z_{n}$

In this case the condition (a), (c), (e) in Theorem 1 is satisfied. From Figures (10)-(12) we can see that $\lim _{n \rightarrow \infty}\left|x_{6 n+j}\right| \rightarrow 0, \lim _{n \rightarrow \infty}\left|y_{6 n+j}\right| \rightarrow \infty, \lim _{n \rightarrow \infty}\left|z_{6 n+j}\right| \rightarrow \infty$.

Example 5. Consider the system (4) with the initial values $x_{-1}=1.3, x_{0}=0.2, y_{-1}=2.5, y_{0}=0.9$, $z_{-1}=1.8, z_{0}=1.1$ and the parameters, $a=15, b=4, c=3, \alpha=0, \beta=5.9, \gamma=6.3$, the following figures represent our solutions,

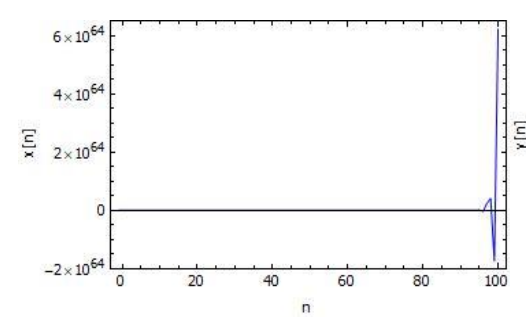

Figure 13. Plots of $x_{n}$

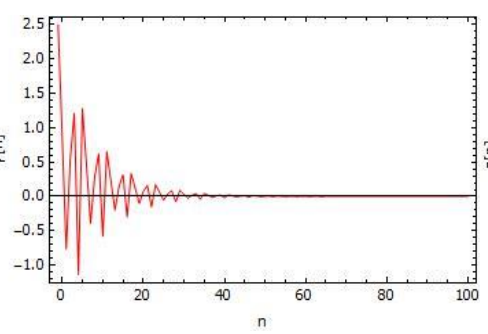

Figure 14. Plots of $y_{n}$

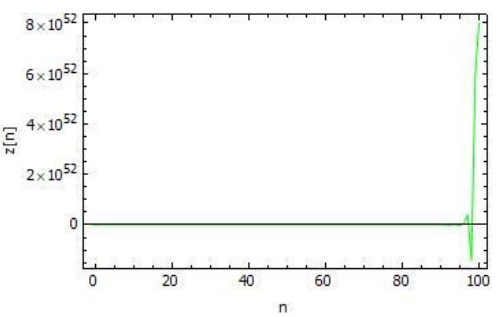

Figure 15. Plots of $z_{n}$

In this case the condition (a), (c), (e) in Theorem 1 is satisfied. From Figures (13)-(15) we can see that $\lim _{n \rightarrow \infty}\left|x_{6 n+j}\right| \rightarrow \infty, \lim _{n \rightarrow \infty}\left|y_{6 n+j}\right| \rightarrow 0, \lim _{n \rightarrow \infty}\left|z_{6 n+j}\right| \rightarrow \infty$.

Example 6. Consider the system (4) with the initial $x_{-1}=1.3, x_{0}=0.2, y_{-1}=2.5, y_{0}=0.9, z_{-1}=$ $1.8, z_{0}=1.1$ and the parameters, $a=3, b=16, c=4, \alpha=2.7, \beta=0, \gamma=7.9$ the solutions are represented by the following figures.

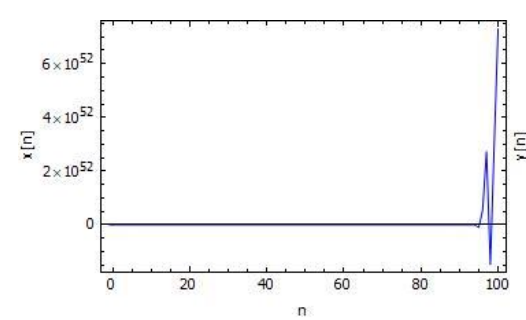

Figure 16. Plots of $x_{n}$

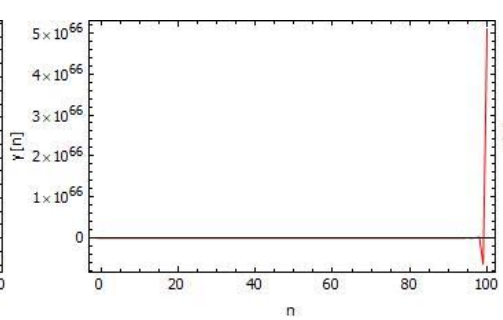

Figure 17. Plots of $y_{n}$

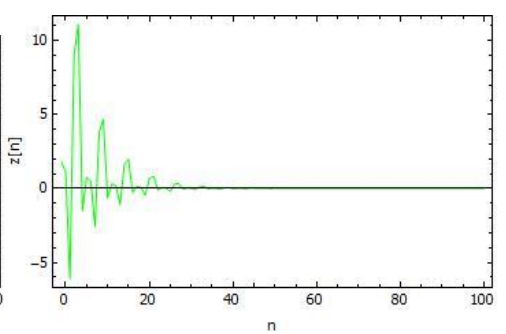

Figure 18. Plots of $z_{n}$

In this case the condition (a), (c), (e) in Theorem 1 is satisfied. From Figures (16)-(18) we can see that $\lim _{n \rightarrow \infty}\left|x_{6 n+j}\right| \rightarrow \infty, \lim _{n \rightarrow \infty}\left|y_{6 n+j}\right| \rightarrow \infty, \lim _{n \rightarrow \infty}\left|z_{6 n+j}\right| \rightarrow 0$.

\section{CONCLUSION}

In this study, we have consider the following three-dimensional system of difference equations $x_{n+1}=\frac{a x_{n} z_{n-1}}{z_{n}-\beta}+\gamma, y_{n+1}=\frac{b y_{n} x_{n-1}}{x_{n}-\gamma}+\alpha, z_{n+1}=\frac{c z_{n} y_{n-1}}{y_{n}-\alpha}+\beta, n \in N_{0}$, 
where the parameters $a, b, c, \alpha, \beta, \gamma$ and the initial values $x_{-i}, y_{-i}, z_{-i}, i=0,1$ are non-zero real numbers.

Firstly we have obtain the closed form of well defined solutions of the aforementioned system using suitable transformation reducing the equations of our system to linear type. Also, we have examine the behavior and the periodicity of the solutions of this system. Finally, numerical examples are provided to support our theoretical results.

It would be interesting to study the $k$ - dimensional version of the system (4).

\section{CONFLICTS OF INTEREST}

No conflict of interest was declared by the authors.

\section{REFERENCES}

[1] Papaschinopoulos, G., Schinas, C.J., Ellina, G., "On the dynamics of the solutions of a biological model”, Journal of Difference Equations and Applications, 20(56): 694-705, (2014).

[2] Yang, K., Cushing, J.M., "Global stability in a nonlinear difference-delay equation model of flour beetle population growth", Journal of Difference Equations and Applications, 2(1): 31-37, (1996).

[3] Gelisken, A., Cinar, C., Yalcinkaya, I., “On a max-type difference equation”, Advances in Difference Equations, 2010(1): 584890, (2010).

[4] Gelisken, A., Cinar, C., Yalcinkaya, I., "On the periodicity of a difference equation with maximum", Discrete Dynamics in Nature and Society, 2008: (2008).

[5] Öcalan, Ö., "Oscillation of nonlinear difference equations with several coefficients", Communications in Mathematical Analysis, 4 (1): 35-44, (2008).

[6] Rabago, J.F.T., "On second-order linear recurrent functions with period $\mathrm{k}$ and proofs to two conjectures of sroysang", Hacettepe Journal of Mathematics and Statistics, 45(2): 429-446, (2016).

[7] Cinar, C., "On the positive solutions of the difference equation $x_{n+1}=\frac{x_{n-1}}{1+x_{n} x_{n-1}}$ ", Applied Mathematics and Computation, 150(1): 21-24, (2004).

[8] Cinar, C., Toufik, M., Yalcinkaya, I., "On the difference equation of higher order", Utilitas Mathematica, 92: 161-166, (2013).

[9] Din, Q., "Global behavior of a rational difference equation", Acta Universitatis Apulensis, 34: 35-49, (2013).

[10] Elmetwally, M.E., Elsayed, E.M., "Dynamics of a rational difference equation", Chinese Annals of Mathematics Series. B, 30B(2): 187-198, (2009).

[11] Elmetwally, H., Yalcinkaya, I., Cinar, C., "On the dynamics of a recursive sequence", Electronic Journal of Mathematical Analysis and Applications, 5(1): 196-201, (2017).

[12] Elsayed, E.M., "On the solutions and periodic nature of some systems of difference equations", International Journal of Biomathematics, 7(6): 1-26, (2014).

[13] Elsayed, E.M., "Solution for systems of difference equations of rational form of order two", International Journal of Computational and Applied Mathematics, 33: 751-765, (2014). 
[14] Elsayed, E.M., "Expression and behavior of the solutions of some rational recursive sequences", Mathematical Methods in the Applied Sciences, 39: 5682-5694, (2016).

[15] Gümüs, M., Soykan, Y., "Global character of a six-dimensional nonlinear system of difference equations", Discrete Dynamics in Nature and Society, 2016: (2016).

[16] Kara, M., Yazlik, Y., "Solvability of a system of non-linear difference equations of higher order", Turkish Journal of Mathematics, 43(3): 1533-1565, (2019).

[17] Kara, M., Yazlik, Y., Tollu, D.T., "Solvability of a system of higher order nonlinear difference equations", Hacettepe Journal of Mathematics and Statistics, doi:10.15672/HJMS.xx (2020).

[18] Kurbanli, A.S., Yalcinkaya, I., Gelisken, A., "On the behavior of the solutions of the system of rational difference equations", International Journal of Physical Sciences, 8(2): 51-56, (2013).

[19] Kurbanli, A.S., Cinar, C., Yalcinkaya, I., "On the behavior of positive solutions of the system of rational difference equations $x_{n+1}=\frac{x_{n-1}}{y_{n} x_{n-1}+1}, y_{n+1}=\frac{y_{n-1}}{x_{n} y_{n-1}+1}$, Mathematical and Computer Modelling, 53: 1261-1267, (2011).

[20] Okumus, I., Soykan, Y., "Dynamical behavior of a system of three-dimensional nonlinear difference equations", Advances in Difference Equations, 2018(223): 1-15, (2018).

[21] Rabago, J.F.T., "On second-order linear recurrent homogeneous differential equations with period $k$ ", Hacettepe Journal of Mathematics and Statistics, 43(6): 923-933, (2014).

[22] Rabago, J.F.T., Bacani, J.B., “On a nonlinear difference equations”, Dynamics of Continuous, Discrete \& Impulsive Systems. Series A. Mathematical Analysis, 24: 375-394, (2017).

[23] Tollu, D.T., Yazlik, Y., Taskara, N., "On fourteen solvable systems of difference equations", Applied Mathematics and Computation, 233: 310-319, (2014).

[24] Tollu, D.T., Yazlik, Y., Taskara, N., "Behavior of positive solutions of a difference equation", Journal of Applied Mathematics \& Informatics, 35: 217-230, (2017).

[25] Touafek, N., "On a second order rational difference equation", Hacettepe Journal of Mathematics and Statistics, 41: 867-874, (2012).

[26] Touafek, N., Elsayed, E.M., "On the periodicity of some systems of nonlinear difference equations", Bulletin Math'ematique de la Soci'et'e des Sciences Math'ematiques de Roumanie, 55(103): 217224, (2012).

[27] Touafek, N., Elsayed, E.M., "On a second order rational systems of difference equations", Hokkaido Mathematical Journal, 44: 29-45, (2015).

[28] Yalcinkaya, I., Cinar, C., "Global asymptotic stability of a system of two nonlinear difference equations", Fasciculi Mathematici, 43: 171-180, (2010).

[29] Yalcinkaya, I., Tollu, D.T., "Global behavior of a second order system of difference equations", Advanced Studies in Contemporary Mathematics, 26(4): 653-667, (2016).

[30] Yazlik, Y., "On the solutions and behavior of rational difference equations", Journal of Computational Analysis and Applications, 17(3): 584-594, (2014). 
[31] Yazlik, Y., Elsayed, E.M., Taskara, N., "On the behaviour of the solutions the solutions of difference equation system", Journal of Computational Analysis and Applications, 16(5): 932-941, (2014).

[32] Yazlik, Y., Tollu, D.T., Taskara, N., "On the behaviour of solutions for some systems of difference equations", Journal of Computational Analysis and Applications, 18(1): 166-178, (2015).

[33] Yazlik, Y., Tollu, D.T., Taskara, N., "On the solutions of a three-dimensional system of difference equations", Kuwait Journal of Science, 43(1): 95-111, (2015).

[34] Yazlik, Y., Kara, M., “On a solvable system of difference equations of fifth-order”, Eskişehir Technical University Journal of Science and Technology B-Theoretical Sciences, 7(1): 29-45, (2019).

[35] Wang, X., Li, Z., "Global asymptotic stability for two kinds of higher order recursive sequences", Journal of Difference Equations and Applications, 22(10): 1542-1553, (2016).

[36] Elabbasy, E.M., El-Metwally, H., Elsayed, E.M., "Qualitative behavior of higher order difference equation", Soochow Journal of Mathematics, 33: 861-873, (2007).

[37] Haddad, N., Touafek, N., Rabago, J.F.T., "Well-defined solutions of a system of difference equations", Journal of Applied Mathematics and Computing, 56(1-2): 439-458, (2018).

[38] Yazlik, Y., Kara, M., "On a solvable system of difference equations of higher-order with period two coefficients", Communications de la Facult'e des Sciences de l'Universit'e d'Ankara S'eries A1. Mathematics and Statistics, 68(2): 1675-1693, (2019).

[39] Elaydi, S., “An Introduction to Difference Equations”. New York: Springer, (1996). 